\title{
Article
}

\section{TwinLIFE: The Twin Longitudinal Investigation of FEtal Discordance}

\author{
Sophie G. Groene ${ }^{1,2}$, Pia Todtenhaupt ${ }^{1,2}$, Erik W. van Zwet ${ }^{3}$, Melissa van Pel ${ }^{4}$, Romy J. M. Berkhout ${ }^{1}$, Monique C. Haak ${ }^{5}$, \\ Arno A. W. Roest ${ }^{6}$, Enrico Lopriore ${ }^{1}$, Jeanine M. M. van Klink ${ }^{1}$ and Bastiaan T. Heijmans ${ }^{2}$ \\ ${ }^{1}$ Neonatology, Department of Pediatrics, Leiden University Medical Center, Leiden, The Netherlands, ${ }^{2}$ Molecular Epidemiology, Department of Biomedical Data \\ Sciences, Leiden University Medical Center, Leiden, The Netherlands, ${ }^{3}$ Medical Statistics, Department of Biomedical Data Sciences, Leiden University Medical \\ Center, Leiden, The Netherlands, ${ }^{4}$ Center for Stem Cell Therapy, Department of Immunohematology and Blood Transfusion, Leiden University Medical Center, \\ Leiden, The Netherlands, ${ }^{5}$ Fetal Medicine, Department of Obstetrics, Leiden University Medical Center, Leiden, The Netherlands and ${ }^{6}$ Pediatric Cardiology, \\ Department of Pediatrics, Leiden University Medical Center, Leiden, The Netherlands
}

\begin{abstract}
Lifelong health is thought to be partially set during intrauterine life by persistent epigenetic changes induced by the prenatal environment. To evaluate this hypothesis, we initiated a prospective longitudinal study in monochorionic (MC) twins: the TwinLIFE study. MC twins are monozygotic, thus in origin genetically identical, and share a single placenta. Although MC twins have many environmental factors in common, in one-third of the MC twin pairs, one fetus has significantly less access to nutrients and resources during pregnancy than its co-twin often resulting in a significant discordance in prenatal growth. Hence, MC twins constitute a unique natural experiment to study the influence of the prenatal environment on health. In TwinLIFE, we will chart intrapair differences in DNA methylation focusing on mesenchymal stromal cells isolated from cord as an advanced proxy of epigenetic dysregulation relevant for long-term health consequences. Next, we will follow up the MC twins for growth, cardiovascular and neurodevelopmental outcomes during childhood and evaluate the impact of an epigenetic signature at birth on future health. The current target is to include $100 \mathrm{MC}$ twin pairs, but we aim to continue enrollment after procuring additional funding. TwinLIFE will not only address an unmet clinical need in the high-risk group of MC twins, but may also advance early-life strategies to prevent adverse growth, cardiovascular and neurodevelopmental outcomes in the general population.
\end{abstract}

Keywords: Monochorionic twins; cardiovascular disease; neurodevelopmental impairment; mesenchymal stromal cells; epigenetics; developmental origins of health and disease

(Received 31 May 2019; accepted 10 June 2019; First Published online 25 July 2019)

\section{Background}

Lifelong cardiovascular and neurodevelopmental risk may be partially set before birth. A low birth weight, preterm birth and prenatal malnutrition are associated with dyslipidemia, adiposity, type 2 diabetes, cardiovascular parameters and neurodevelopmental disorders (Barker, 2006; Gluckman et al., 2008; Lumey et al., 2011). The common denominator of these factors is that they represent an adverse intrauterine environment, resulting in greater long-term health risk.

Despite research dating back as early as the 1970s, the molecular mechanism underlying these findings remains to be resolved. Epigenetic mechanisms are widely perceived to be a strong candidate (Waterland \& Michels, 2007). Epigenetic marks, such as DNA methylation, are primarily set during intrauterine development (Guo et al., 2014; Slieker et al., 2015) and, in conjunction with transcription factors, control the regulation of gene expression. Experimental perturbation of the intrauterine environment in animal models can induce persistent epigenetic changes at the level

Author for correspondence: Bastiaan T. Heijmans, Email: b.t.heijmans@lumc.nl Cite this article: Groene SG, Todtenhaupt P, van Zwet EW, van Pel M, Berkhout RJM, Haak MC, Roest AAW, Lopriore E, van Klink JMM, and Heijmans BT. (2019) TwinLIFE: The Twin Longitudinal Investigation of FEtal Discordance. Twin Research and Human Genetics 22: 617-622, https://doi.org/10.1017/thg.2019.38 of DNA methylation (Waterland \& Jirtle, 2003). Studies in humans included those on the Dutch Hunger Winter, a severe famine at the end of World War II (Heijmans et al., 2008; Tobi et al., 2009). Individuals exposed to prenatal famine were found to have accumulated DNA methylation differences near genes involved in growth and development (Tobi et al., 2014). Specific DNA methylation differences were observed to mediate the association of prenatal famine and adult BMI and blood lipids (Tobi et al., 2018).

However, despite such promising findings, it can be argued that the field still has to deliver robust mechanistic insight and early-life epigenetic biomarkers of long-term health outcomes. This may be achieved by focusing on defined present-day populations experiencing severe intrauterine adversity. Study designs that enable strong inference, tissues other than peripheral blood, and longitudinal analyses starting before birth can be used to enable the testing of epigenetic mediation of prenatal adversity in later health.

Therefore, we established TwinLIFE, a prospective longitudinal investigation of fetal discordance within monochorionic (MC) twin pairs, who are monozygotic twins sharing a single placenta. MC twins can be considered a unique natural experiment as they in origin have the same genetic makeup and share many environmental factors, from the womb, age and sex to a range of maternal

(C) The Author(s) 2019. This is an Open Access article, distributed under the terms of the Creative Commons Attribution licence (https://creativecommons.org/licenses/by/4.0/), which permits unrestricted re-use, distribution, and reproduction in any medium, provided the original work is properly cited. 
and paternal factors. Yet, they are frequently exposed to a vastly discordant prenatal environment due to complications that result from sharing a single placenta. In this study, we will follow the prenatal development of MC twins, store biological samples at birth and follow up the twins into childhood. Our aim is to evaluate whether DNA methylation differences in mesenchymal stromal cells (MSCs) isolated from cord (as a proxy for somawide epigenetic changes) mediate the impact of prenatal adversity on growth, cardiovascular and neurodevelopmental outcomes. Moreover, we will collect buccal swabs to evaluate whether the observed epigenetic changes in the MSCs are also represented in peripheral tissue. The results will be of immediate relevance to MC twins, but the impact may extend to the general population of singletons experiencing severe pregnancy complications such as fetal growth restriction. The model of MC twins may help unravel the pathophysiological mechanisms linking an adverse intrauterine environment to lifelong growth patterns, cardiovascular disease (CVD) and neurodevelopmental impairment (NDI) risk.

\section{Hypothesis and Objectives}

The overall hypothesis is that MC twin pairs who are discordant for intrauterine adversity display a distinct epigenetic signature in the MSCs at birth which, in turn, explain future interindividual variation in cardiac and vascular measures of CVD, neurodevelopmental outcomes, and growth patterns. We expect that testing this hypothesis will pinpoint the biological pathways that set long-term health risks when epigenetically changed during fetal development.

To test this hypothesis, the objectives of TwinLIFE are:

1. To establish a longitudinal MC twin cohort with prospectively recorded data on growth, cardiovascular and neurodevelopmental parameters measured pre- and perinatally and at 2 , 5 and 8 years of follow-up to quantify the discordance in the prenatal environment and health outcomes.

2. To define epigenetic changes induced by an adverse intrauterine environment in the MSCs from umbilical cord by conducting a within-pair comparison of genomewide DNA methylation.

3. To evaluate the link between the observed epigenetic alterations and growth, cardiovascular, and neurodevelopmental health outcomes assessed at birth and 2, 5 and 8 years of follow-up.

\section{Study Procedures}

\section{Establishing the MC Twin Cohort}

The Leiden University Medical Center (LUMC) is the national referral center for complicated MC twin pregnancies in the Netherlands. The LUMC provides top-referent care that includes close monitoring of development and health of MC twins from early pregnancy to childhood. Therefore, a large proportion of clinical data available for TwinLIFE is routinely collected as part of standard care. In the LUMC, approximately $1 \mathrm{MC}$ twin pair is born per week ensuring a steady enrollment of MC twins into TwinLIFE. We aim to enroll at least $100 \mathrm{MC}$ twin pairs over a period of 2-3 years. Provided that we successfully acquire additional funding, we will increase our sample size beyond 100 pairs. Twins will be followed throughout childhood. This includes the time from inclusion (from week 14 of gestation to birth) to the completion of the follow-up assessments at 2, 5 and 8 years (Figure 1).
Monochorionicity is diagnosed with ultrasound in the first trimester of gestation, a procedure undergone by all pregnant women in the Netherlands. Women carrying an MC twin are referred to a hospital, either a general hospital or a university medical center, for close monitoring of the pregnancy which consists of an ultrasound every other week for the early detection of amniotic fluid discordance, differences in growth or other complications. If complications are detected, women are referred to a university medical center for specialist care. MC twin pregnancies are at risk of developing numerous complications, regularly in combination, among which selective fetal growth restriction (sFGR), twin-twin transfusion syndrome (TTTS) and twin anemia-polycythemia sequence (TAPS) represent the large majority. sFGR (occurring in up to $25 \%$ of MC twins) is characterized by unequal placenta sharing and fetal growth discordance as illustrated in Figure 2 (Inklaar et al., 2014). TTTS (occurring in up to $10 \%$ of MC twins) results from severe intertwin blood flow imbalances and is diagnosed based on discordancy in amniotic fluid volume (twin oligo-polyhydramnios sequence) (Lewi et al., 2008). Lastly, TAPS (occurring up to $5 \%$ of MC twins) is caused by chronic blood flow imbalances through minuscule vascular anastomoses and diagnosed based on large hemoglobin discordance before or at birth (Slaghekke et al., 2010).

Written informed consent from both parents $(\geq 18$ years and able to consent) is a prerequisite before entering TwinLIFE. The following cases will be excluded from the study: twins with major anatomical abnormalities (interference with outcomes), triplet or higher order multiple pregnancies (different population, cannot be compared to MC twins), cases with Twin Reversed Arterial Perfusion (TRAP) sequence (acardiac twin) and/or cases with single or double fetal demise (no intertwin comparison possible after birth).

We will conduct a comprehensive series of pre- and perinatal assessments (Tables 1 and 2), which consist of a series of assessments part of standard care supplemented with a fetal cardiac functioning measurement, a postnatal cardiac ultrasound and questionnaires specific for the study. Prenatally, fetal growth and cardiac functioning will be recorded using detailed ultrasound scans every 2 weeks or more frequently on clinical indication. Furthermore, maternal and paternal characteristics will be recorded prenatally using a questionnaire, which collects information on demographics, medication and nutritional supplement use, intoxications and medical history. Also, parental wellbeing will be assessed using the Brief Symptom Inventory (BSI) (Lang, 2003) for quantification of somatization, depression and anxiety. After birth, we will assess anthropometric measurements (birth weight, length in supine position and head circumference), brain and cardiac development. Placental characteristics will be recorded after color dye injection by trained staff according to our routine clinical program for placenta analysis in MC twins for in-depth analysis of the placental angioarchitecture (Lopriore et al., 2011). Common neonatal morbidities will be recorded from birth and admission to the neonatal intensive care unit until discharge to home by the treating neonatologist. At 6 months (corrected for prematurity), parental wellbeing will be reevaluated using the BSI and child nutrition will be recorded using a standardized questionnaire.

\section{Defining Epigenetic Changes at Birth}

At birth, we will collect umbilical cord tissue, cord blood and buccal swabs for the TwinLIFE Biobank. Umbilical cords will be 


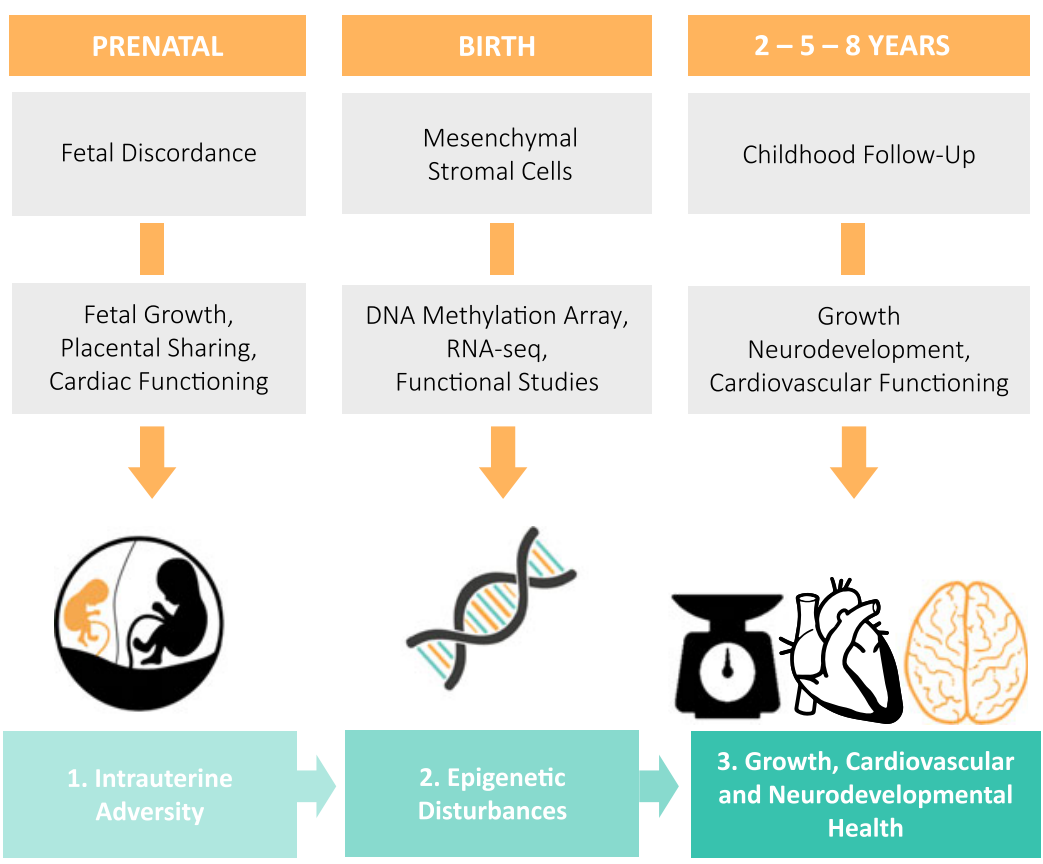

Fig. 1. An overview of the study design and the main procedures that subjects will undergo over the course of the study.
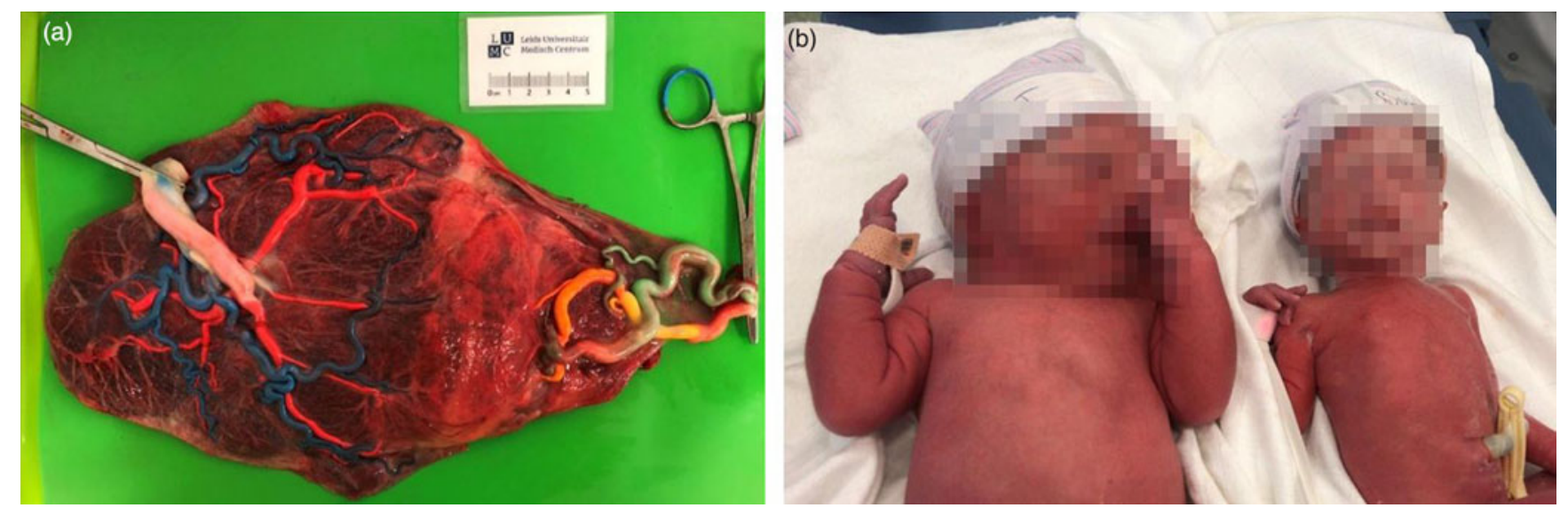

Fig. 2. Selective fetal growth restriction: (a) an injected placenta demonstrating an unequal placental share division (79\% vs. $21 \%$ ) and a laser demarcation line following fetoscopic laser surgery for coexistent TTTS; (b) an MC twin pair affected by selective fetal growth restriction treated at the LUMC (gestational age at birth 32 weeks, birth weights 2300 and $1200 \mathrm{~g}$ ). The larger placental share (a, left) belonged to the larger co-twin (b, left).

collected in a sterile PBS-antibiotic/antimycotic solution on birth as a source of MSCs. MSCs are cells of mesodermal origin with the capacity to differentiate into multiple cell types including osteoblasts, adipocytes and chondroblasts using specific culture conditions (Swamynathan et al., 2014; van der Garde et al., 2015). MSCs can be obtained from umbilical cord without any risk for the donor and expanded to high numbers for experiments. For MSC isolation, a modified explant method operational at the LUMC will be adapted and standardized (van der Garde et al., 2015). After expansion for approximately 10 days, the explants are removed and the MSCs are transferred into culture flasks. The transferred cells will be grown to $>70 \%$ confluence, frozen and stored until further expansion is required. Subsequent MSC expansions of MSCs obtained from co-twins will be performed simultaneously to minimize batch effects. The uniform classification system for MSCs, introduced by the International Society of Cellular Therapy, will be adopted to ensure unambiguous identification and separation of MSCs (Dominici et al., 2006). The number of cells after expansion will be sufficient for the epigenetic profiling, RNA expression analysis, cellular phenotyping, and functional differentiation studies.

Cord blood will be collected in sterile blood collection tubes, containing a citrate phosphate dextrose adenine (CPDA) solution. Subsequently, mononuclear cells and plasma will be isolated using a Ficoll density gradient. The isolated cells and plasma will be stored for further experiments, such as DNA methylation analysis of specific cells types. Buccal swabs will be collected, postnatally for DNA extraction and DNA methylation analysis.

We will perform genomewide DNA methylation profiling of MSCs from the twins, using the Illumina EPIC methylation array (Zhou et al., 2017). The platform provides a cost-effective coverage of $\sim 850,000$ potentially methylated CG dinucleotides across the genome and is enriched for regions known to be involved in genomic regulation. The EPIC array is the successor of the $450 \mathrm{k}$ 
Table 1. Timing and goal of assessments of TwinLIFE

\begin{tabular}{|c|c|c|}
\hline Timing & Assessment & Goal \\
\hline $\begin{array}{l}\text { Prenatal } \\
\text { 1st trim-delivery }\end{array}$ & Ultrasound/2 wks & $\begin{array}{l}\text { To document fetal growth, cardiac/brain development and cardiac functioning; } \\
\text { to quantify prenatal growth discordance }\end{array}$ \\
\hline \multirow{6}{*}{$\begin{array}{l}\text { Birth } \\
\text { Delivery-1 }{ }^{\text {st }} \text { wk }\end{array}$} & Cord, cord blood & To collect MSCs for epigenetic studies \\
\hline & Buccal swab & To examine the persistence of epigenetic changes in peripheral tissue \\
\hline & Placental evaluation & $\begin{array}{l}\text { To analyze placental angioarchitecture, including placental share, cord } \\
\text { insertion, incidence, type and size of vascular anastomoses }\end{array}$ \\
\hline & Birth weight & To quantify postnatal growth discordance \\
\hline & Cranial ultrasound & $\begin{array}{l}\text { To assess brain size and shape of brain structures, maturation and type of } \\
\text { cerebral injury }\end{array}$ \\
\hline & Cardiac ultrasound & $\begin{array}{l}\text { To determine cardiovascular size and function as assessed by left ventricular } \\
\text { mass, left atrial diameter, aortic root diameter, shortening fraction and myocardial } \\
\text { deformation }\end{array}$ \\
\hline \multirow[t]{5}{*}{$\begin{array}{l}\text { Follow-up } \\
2,5 \text { and } 8 \text { years }\end{array}$} & $\begin{array}{l}\text { Height, weight and head circumference } \\
\text { measurements }\end{array}$ & To document infant growth \\
\hline & $\begin{array}{l}\text { Formal neurological examination and } \\
\text { standardized psychometric tests (Bayley } \\
\text { scales at } 24 \text { months, Wechsler scales at } \\
5 \text { and } 8 \text { years) }\end{array}$ & $\begin{array}{l}\text { To examine neurodevelopmental outcome, including cognitive and motor } \\
\text { development }\end{array}$ \\
\hline & CBCL, Lexi lijst, PedsQoL, BRIEF & To assess quality of life, language and behavioral development of the twins \\
\hline & IBQ-R, ECBQ, CBQ, AISI & To evaluate temperament and attachment of the twins \\
\hline & Cardiac ultrasound, aPWV, cIMT & $\begin{array}{l}\text { To determine CVD risk by focusing on three key areas: cardiac dimensions and } \\
\text { load (left ventricular mass and left-sided structures), vascular stiffness (aPWV) and } \\
\text { remodeling of the arterial wall (cIMT) }\end{array}$ \\
\hline
\end{tabular}

Note: trim = trimester, wks = weeks, MSCs = mesenchymal stromal cells, CBCL = Child Behavior Checklist, PedsQoL = Pediatric Quality of Life Inventory, BRIEF = Behavior Rating Inventory of Executive Functioning, Infant Behavior Questionnaire, ECBQ = Early Childhood Behavior Questionnaire, CBQ = Children's Behavior Questionnaire, AISI = Attachment Insecurity Screening Inventory, $\mathrm{aPWV}=$ aortic pulse wave velocity, $\mathrm{CIMT}=$ carotid intima-media thickness, $\mathrm{CVD}=$ cardiovascular disease.

array that has been widely used to successfully identify epigenetic changes after adverse prenatal exposures, including by ourselves in the context of the Dutch Hunger Winter (Tobi et al., 2015). We will use our established work flow for quality control, normalization and analysis methylation array data, which is publicly available (https://github.com/molepi/DNAmArray) and combines our own BioConductor packages with the best practices in the field (van Iterson et al., 2014, 2017, 2018). Co-twins will be measured on the same array and array row and will be randomized with respect to array column.

\section{Uncovering Epigenetic Influences on Growth, CVD and NDI Risk}

To study the associations of epigenetic changes at birth with precursors of CVD and NDI risk in childhood, we will initiate a follow-up examination of MC twins at least 2 years of age aimed at a focused growth, cardiac, vascular and neurodevelopmental characterization. Parents and their MC twins will be invited to the LUMC for follow-up examinations at the age of 2, 5 and 8 years (at term equivalent age). Transthoracic echocardiography will be used to assess differences in structural and functional cardiac measures (Toemen et al., 2017). Furthermore, aortic pulse-wave velocity (PWV) reflecting vascular stiffness will be assessed using aortic length regression equation based on the subject's height and two pulsed Doppler recordings of the aorta (Jo et al., 2010). Measurement of the intima-media thickness (IMT) of the carotid artery, demonstrating the remodeling of the arterial wall, is feasible in small children and will be measured with ultrasonography with a linear array probe of 7.5 MHz (Dilli et al., 2017).
Long-term follow-up of complicated MC twin pregnancies is part of our standard clinical care at the LUMC and includes a formal neurological examination (Touwen et al., 1992), measurement of relevant growth parameters, measurement of blood pressure and an assessment of cognitive and motor development using standardized psychometric tests (van Klink et al., 2015, 2016). At the (term equivalent) age of 24 months, cognitive and motor development will be assessed using Bayley scales (Bayley, 2006). At 5 and 8 years of follow-up, cognitive development will be assessed using Wechsler scales (Wechsler, 2002, 2014). During the visit, we will also collect anthropometric data through our national system of primary care physicians who monitor growth in every Dutch child throughout the first 2 years of life.

To assess quality of life, language and behavioral development of the twins, online questionnaires will be filled in by the parents using a web-based application (Baron, 2000; Varni et al., 2001). These questionnaires are part of our standard clinical care. In addition, we will evaluate temperament and attachment using validated questionnaires (Putnam et al., 2006; Spruit et al., 2018) (Table 2).

\section{Future Plans}

As of January 2019, we have started the inclusion of twin pairs into TwinLIFE. We aim to create a dynamic cohort, where we continuously include new MC twins to increase the sample size beyond 100 pairs and also extend the follow-up beyond 8 years to further deepen our understanding of the effect of an adverse intrauterine environment on growth, CVD and NDI risk at a later ages as mediated by epigenetic alterations. We intend to broaden the scope of our research by adding other disease areas (e.g. lung disease) and 
Table 2. Overview of evaluations and measurements within TwinLIFE

\begin{tabular}{|c|c|}
\hline \multicolumn{2}{|c|}{ I. Establishing a longitudinal MC twin cohort and quantifying prenatal discordance } \\
\hline Prenatal & $\begin{array}{l}\text { - Ultrasound (every } 2 \text { weeks) for monitoring fetal growth, cardiac and brain development and diagnosing complications } \\
\text { - Additional cardiac measurements performed during routine ultrasound: tricuspid, mitral, aortic and pulmonary valve, } \\
\text { speckle-tracking strain analysis left and right ventricle, color tissue Doppler imaging of myocardium } \\
\text { - Questionnaires }\end{array}$ \\
\hline Birth & $\begin{array}{l}\text { Neonatal evaluation (birth weight, head circumference, length in supine position), cranial ultrasound, echocardiography } \\
\text { including cardiac functioning, placental examination }\end{array}$ \\
\hline Biobank & Cord blood, cord, buccal swab \\
\hline Epigenetics & Illumina Epic 850k DNA methylation array, gPCR \\
\hline Cellular & Metabolic characterization \\
\hline \multicolumn{2}{|c|}{ III. Uncovering epigenetic influences on CVD and NDI risk } \\
\hline $\begin{array}{l}\text { Follow-up 2, } 5 \text { and } \\
8 \text { years }\end{array}$ & Growth, neurodevelopment, behavior, temperament and attachment, echocardiography, cIMT, aPWV, buccal swab \\
\hline
\end{tabular}

Note: $\mathrm{MC}=$ monochorionic, $\mathrm{MSC}=$ mesenchymal stromal cells, $\mathrm{qPCR}=$ quantitative polymerase chain reaction, $\mathrm{CVD}=$ cardiovascular disease, $\mathrm{NDI}=$ neurodevelopmental impairment, cIMT $=$ carotid intima-media thickness, aPWV $=$ aortic pulse-wave velocity

analyzing other cell types than cord MSCs (e.g. immune cells and endothelial progenitor cells (EPCs)). Finally, we are committed to collaborate with other MSC-focused cohorts, twin registries and birth cohorts.

Acknowledgments. We wish to thank the mothers and fathers for participating in TwinLIFE with their newborn twins.

Financial support. TwinLIFE is funded by an Established Investigator grant from the Dutch Heart Foundation (2017T075). The funders had no role in study design, data collection, analysis, decision to publish or preparation of the manuscript.

\section{Conflict of interest. None.}

Ethical standards. The authors assert that all procedures contributing to this work comply with the ethical standards of the relevant national and institutional committees on human experimentation and with the Helsinki Declaration of 1975 , as revised in 2008 .

\section{References}

Barker, D. J. (2006). Adult consequences of fetal growth restriction. Clinical Obstetrics and Gynecology, 49, 270-283.

Baron, I. S. (2000). Behavior rating inventory of executive function. Child Neuropsychology, 6, 235-238.

Bayley, N. (2006). Bayley scales of infant and toddler development (3rd ed.). San Antonio, TX: Pearson Education, Inc.

Dilli, D., Ozkan, E., Ozkan, M. B., Aydin, B., Ozyazici, A., Fettah, N., \& Okumus, N. (2017). Umbilical cord asymmetric dimethylarginine levels and ultrasound assessment of carotid arteries in neonates born small for gestational age. Journal of Maternal-Fetal and Neonatal Medicine, 30, 492-496.

Dominici, M., Le Blanc, K., Mueller, I., Slaper-Cortenbach, I., Marini, F., Krause, D., \& Horwitz, E. (2006). Minimal criteria for defining multipotent mesenchymal stromal cells. The International Society for Cellular Therapy position statement. Cytotherapy, 8, 315-317.

Gluckman, P. D., Hanson, M. A., Cooper, C., \& Thornburg, K. L. (2008). Effect of in utero and early-life conditions on adult health and disease. New England Journal of Medicine, 359, 61-73.

Guo, H., Zhu, P., Yan, L., Li, R., Hu, B., Lian, Y., \& Qiao, J. (2014). The DNA methylation landscape of human early embryos. Nature, 511, 606-610.

Heijmans, B. T., Tobi, E. W., Stein, A. D., Putter, H., Blauw, G. J., Susser, E. S., \& Lumey, L. H. (2008). Persistent epigenetic differences associated with prenatal exposure to famine in humans. Proceedings of the National Academy of Sciences of the United States of America, 105, 17046-17049.
Inklaar, M. J., van Klink, J. M., Stolk, T. T., van Zwet, E. W., Oepkes, D., \& Lopriore, E. (2014). Cerebral injury in monochorionic twins with selective intrauterine growth restriction: a systematic review. Prenatal Diagnosis, 34, 205-213.

Jo, C. O., Lande, M. B., Meagher, C. C., Wang, H., \& Vermilion, R. P. (2010). A simple method of measuring thoracic aortic pulse wave velocity in children: methods and normal values. Journal of the American Society of Echocardiography, 23, 735-740.

Lang, A. J. (2003). Brief intervention for co-occurring anxiety and depression in primary care: a pilot study. International Journal of Psychiatry in Medicine, $33,141-154$.

Lewi, L., Jani, J., Blickstein, I., Huber, A., Gucciardo, L., Van Mieghem, T., \& Deprest, J. (2008). The outcome of monochorionic diamniotic twin gestations in the era of invasive fetal therapy: a prospective cohort study. American Journal of Obstetrics and Gynecology, 199, 514.e1-514.e8.

Lopriore, E., Slaghekke, F., Middeldorp, J. M., Klumper, F. J., van Lith, J. M., Walther, F. J., \& Oepkes, D. (2011). Accurate and simple evaluation of vascular anastomoses in monochorionic placenta using colored dye. Journal of Visualized Experiments 5, e3208.

Lumey, L. H., Stein, A. D., \& Susser, E. (2011). Prenatal famine and adult health. Annual Review of Public Health, 32, 237-262.

Putnam, S. P., Gartstein, M. A., \& Rothbart, M. K. (2006). Measurement of fine-grained aspects of toddler temperament: The early childhood behavior questionnaire. Infant Behavior and Development, 29, 386-401.

Slaghekke, F., Kist, W. J., Oepkes, D., Pasman, S. A., Middeldorp, J. M., Klumper, F. J., \& Lopriore, E. (2010). Twin anemia-polycythemia sequence: Diagnostic criteria, classification, perinatal management and outcome. Fetal Diagnosis and Therapy, 27, 181-190.

Slieker, R. C., Roost, M. S., van Iperen, L., Suchiman, H. E., Tobi, E. W., Carlotti, F., \& Chuva de Sousa Lopes, S. M. (2015). DNA methylation landscapes of human fetal development. PLoS Genetics, 11, e1005583.

Spruit, A., Wissink, I., Noom, M. J., Colonnesi, C., Polderman, N., Willems, L., \& Stams, G. (2018). Internal structure and reliability of the Attachment Insecurity Screening Inventory (AISI) for children age 6 to 12. BMC Psychiatry, 18, 30.

Swamynathan, P., Venugopal, P., Kannan, S., Thej, C., Kolkundar, U., Bhagwat, S., \& Balasubramanian, S. (2014). Are serum-free and xeno-free culture conditions ideal for large scale clinical grade expansion of Wharton's jelly derived mesenchymal stem cells? A comparative study. Stem Cell Research \& Therapy, 5, 88.

Tobi, E. W., Goeman, J. J., Monajemi, R., Gu, H., Putter, H., Zhang, Y., \& Heijmans, B. T. (2014). DNA methylation signatures link prenatal famine exposure to growth and metabolism. Nature Communications, 5,5592 . 
Tobi, E. W., Lumey, L. H., Talens, R. P., Kremer, D., Putter, H., Stein, A. D., \& Heijmans, B. T. (2009). DNA methylation differences after exposure to prenatal famine are common and timing- and sex-specific. Human Molecular Genetics, 18, 4046-4053.

Tobi, E. W., Slieker, R. C., Luijk, R., Dekkers, K. F., Stein, A. D., Xu, K. M., \& Heijmans, B. T. (2018). DNA methylation as a mediator of the association between prenatal adversity and risk factors for metabolic disease in adulthood. Science Advances, 4, eaao4364.

Tobi, E. W., Slieker, R. C., Stein, A. D., Suchiman, H. E., Slagboom, P. E., van Zwet, E. W., \& Lumey, L. H. (2015). Early gestation as the critical timewindow for changes in the prenatal environment to affect the adult human blood methylome. International Journal of Epidemiology, 44, 1211-1223.

Toemen, L., Gaillard, R., van Osch-Gevers, L., Helbing, W. A., Hofman, A., \& Jaddoe, V. W. (2017). Tracking of structural and functional cardiac measures from infancy into school-age. European Journal of Preventive Cardiology, 24, 1408-1415.

Touwen, B. C., Hempel, M. S., \& Westra, L. C. (1992). The development of crawling between 18 months and four years. Developmental Medicine \& Child Neurology, 34, 410-416.

van der Garde, M., van Pel, M., Millan Rivero, J. E., de Graaf-Dijkstra, A., Slot, M. C., Kleinveld, Y., \& Zwaginga, J. J. (2015). Direct comparison of Wharton's Jelly and bone marrow-derived mesenchymal stromal cells to enhance engraftment of cord blood CD34(+) transplants. Stem Cells and Development, 24, 2649-2659.

van Iterson, M., Cats, D., Hop, P., Consortium, B., \& Heijmans, B. T. (2018). omicsPrint: detection of data linkage errors in multiple omics studies. Bioinformatics, 34, 2142-2143.

van Iterson, M., Tobi, E. W., Slieker, R. C., den Hollander, W., Luijk, R., Slagboom, P. E.,\& Heijmans, B. T. (2014). MethylAid: visual and interactive quality control of large Illumina 450k datasets. Bioinformatics, 30, 3435-3437.

van Iterson, M., van Zwet, E. W., Consortium, B., \& Heijmans, B. T. (2017). Controlling bias and inflation in epigenome- and transcriptome-wide association studies using the empirical null distribution. Genome Biology, $18,19$.

van Klink, J., Koopman, H. M., Middeldorp, J. M., Klumper, F. J., Rijken, M., Oepkes, D.,\& Lopriore, E. (2015). Long-term neurodevelopmental outcome after selective feticide in monochorionic pregnancies. BJOG, 122, $1517-1524$.

van Klink, J. M. M., Slaghekke, F., Balestriero, M. A., Scelsa, B., Introvini, P., Rustico, M., \& Lopriore, E. (2016). Neurodevelopmental outcome at 2 years in twin-twin transfusion syndrome survivors randomized for the Solomon trial. American Journal of Obstetrics and Gynecology, 214, 113.e1-e7.

Varni, J. W., Seid, M., \& Kurtin, P. S. (2001). PedsQL 4.0: reliability and validity of the Pediatric Quality of Life Inventory version 4.0 generic core scales in healthy and patient populations. Medical Care, 39, 800-812.

Waterland, R. A., \& Jirtle, R. L. (2003). Transposable elements: targets for early nutritional effects on epigenetic gene regulation. Molecular and Cellular Biology, 23, 5293-5300.

Waterland, R. A., \& Michels, K. B. (2007). Epigenetic epidemiology of the developmental origins hypothesis. Annual Review of Nutrition, 27, 363-388.

Wechsler, D. (2002). Wechsler preschool and primary scale of intelligence (3rd ed.). (WPPSI-III-NL). TX: The Psychological corporation.

Wechsler, D. (2014). Wechsler intelligence scale for children (5th ed.). San Antonio, TX: Wechsler Intelligence Scale for Children.

Zhou, W., Laird, P. W., \& Shen, H. (2017). Comprehensive characterization, annotation and innovative use of Infinium DNA methylation BeadChip probes. Nucleic Acids Research, 45, e22. 